\title{
Study on Heat Transfer Characteristics of the Whole Plate Fin Tube Cooler
}

\author{
Yuzhu Zhou ${ }^{1}$, Lin Zhang ${ }^{1,2}$, Shi $\mathrm{Bu}^{1,2}$, Cheng $\mathrm{Sun}^{1}$, Weigang $\mathrm{Xu}^{1}$, \\ Yongchang Xiao ${ }^{1,2}$, Lin Liu ${ }^{1,2}$ \\ ${ }^{1}$ School of Mechanical Engineering, Changzhou University, Changzhou, China; \\ ${ }^{2}$ Jiangsu Key Laboratory of Green Process Equipment, Changzhou University, Changzhou, China.
}

Received: 20 January 2020; Received in revised form: 22 April 2020; Accepted: 26 May 2020; Published online 2 June 2020

(C) Published at www.ijtf.org

\begin{abstract}
In order to enhance the cooling efficiency of plate-fin motor cooler, two kinds of plate-fin-tubes were proposed, which named triangle-wing and convex fin-tube. The heat transfer characteristics of fin-tubes are investigated via numerical simulation and experiment. The result showed that the $\mathrm{k}-\varepsilon$ turbulence model is highly accurate in simulation of cooler, and the deviation of average heat transfer coefficient and frictional pressure drop between experiment and simulation is within $10 \%$ and $8 \%$, respectively. Both of the triangle-wing and convex fin-tube can increase the heat transfer efficiency of plate-fin cooler. The frictional pressure drop also has an approximate variation trend. In addition, the pressure drop of convex-fin-tube is smaller than triangle-wing-fin-tube on the premise of the same heat transfer coefficient, so the heat transfer performance of convex-fin-tube is the best.
\end{abstract}

Keywords: Numerical Simulation; Motor Cooling; Plate-Fin Cooler; Heat Transfer Characteristics

\begin{tabular}{|c|c|c|c|}
\hline \multicolumn{4}{|c|}{ Nomenclature } \\
\hline$A$ & heat transfer area & $c_{\mathrm{p} 2}$ & $\begin{array}{l}\text { average constant pressure specific } \\
\text { heat capacity of cold fluid }\end{array}$ \\
\hline$c_{\mathrm{p} 1}$ & $\begin{array}{l}\text { average constant pressure specific heat } \\
\text { capacity of thermal fluid }\end{array}$ & $T_{2}$ & outlet temperature of thermal fluid \\
\hline$T_{1}$ & inlet temperature of thermal fluid & $t_{2}$ & outlet temperature of cold fluid \\
\hline$t_{1}$ & inlet temperature of cold fluid & $q_{\mathrm{m} 2}$ & mass flow of cold fluid \\
\hline$q_{\mathrm{m} 1}$ & mass flow of thermal fluid & $\tau$ & Viscous force \\
\hline$u$ & velocity & $\rho$ & fluid density \\
\hline$\Delta t_{\mathrm{m}}$ & logarithmic mean temperature difference & & \\
\hline
\end{tabular}

Corresponding e-mail: z3821315@yeah.net 
Yuzhu Zhou et al.

International Journal of Thermofluid Science and Technology (2020), Volume 7, Issue 2, Paper No. 070204

\section{Introduction}

Electric motors play a vital role in all industries. However, the main problem of the motor is its heat generation [1]. With the internal or local high temperature, operating conditions will be deteriorated which lead to the safety performance of motor decline [2]. What's worse, more economic losses are generated. Therefore, the common goal of many researchers is to solve the problem of motor temperature rise.

The cooler is the main auxiliary cooling device for the motor. In the same volume, the heat transfer efficiency of fin-tube heat exchanger is higher than ordinary tube-fin because connected fin-to-fin can increase contact area [3], thus, the fin-tube heat exchanger is usually used to cooling motor. At present, structural optimization is a hot research on the heat transfer performance of fin-tube heat exchangers [4]. The heat transfer characteristics of finned-tube heat exchanger can be improved with the help of the vortex generator. Gupta et al. [5] and Lee[6,7] used numerical simulation to investigate the heat transfer performance of a fin-tube heat exchanger with longitudinal vortex generators. They observed that thermohydraulic performance of the finned tube heat exchanger has been significantly improved, even to $71 \%$. Li et al. [8,9] measured and compared the air-side pressure drop and total heat transfer coefficient of finless heat exchangers with or without vortex generators and 5 all-aluminum parallel multi-port heat exchangers. They found that the heat transfer performance of a finless heat exchanger with a longitudinal vortex generator (LVG) is still approximately $40 \%$ less than that of a fin-tube heat exchanger. Salleh et al. [10] explored that the flow characteristics and thermal performance across the fin-tube heat exchangers with and without trapezoidal winglet vortex generator (TWVG). It was found that the arrangement, geometry, aspect ratio and angle of attack of the TWVG that resulted in the widest projected area facing the main flow has provided higher heat transfer enhancement.

Heat transfer characteristics can also be improved by changing the fin structure. According to the geometry of the fins, it may include plain fins, wavy fins [11], offset strip fins, composite fin shapes[12], louvered fins [13], slotted fin surfaces[14], herringbone wavy fins [15], and some enhanced fins [16,17].Wang et al. [18] investigated the heat transfer characteristics of seceral fin-tube heat exchangers, and they found that the heat transfer performance of single H-type finned elliptical tube is better than single H-type finned tube at the same spacing. Pengfei Wang et al. [19] investigated the influence of tube ellipticity ratio $\mathrm{e}$, tube rotation angle $\theta$ and fin spacing $\mathrm{H}$ on heat transfer capability, and come to conclusion that the inclined elliptical fin-tube heat exchanger with the tube ellipticity ratio $\mathrm{e}=0.6$ and rotation angle $\theta=30^{\circ}$ has the best heat exchange performance and airflow characteristics. Li et al. [20] proposed a new type of LVG fin and achieved the overall performance via experiment, and the result showed that it is better than that of wave-shaped fins. Kim [21] developed a newly nonsymmetric slit-finned-tube heat exchangers which had more slits in the second row than the first row. He found that the slit fin heat exchangers show superior heat-transfer characteristics than conventional louver fin or slit fin heat exchangers, especially at the second row. This was attributed to the many narrow slits that formed at the second row, which maintain thin water film along the slits and smooth the condensate flow. Toubiana et al. [22] carried out a three-dimensional numerical simulation of a multi-rows elliptical finned-tube heat exchanger for four Reynolds numbers in the transitional regime by means of highly resolved Large Eddy Simulations. The result showed that the heat transfer coefficient is correlated with the turbulent structures and unsteadiness. 
Yuzhu Zhou et al.

International Journal of Thermofluid Science and Technology (2020), Volume 7, Issue 2, Paper No. 070204

It can be seen that better heat transfer performance can be obtained by changing the fin structure or increasing the degree of turbulence. Therefore, according to the current mainstream research direction, combined with the research needs of enterprises for actual plate-shaped fin coolers, a new and efficient plat- fin cooling unit with triangle-wing-fin is proposed in this paper. This kind of fin can not only increase the heat transfer area but also increase the turbulence degree. The heat transfer performance of three finned tube coolers was compared and analyzed by changing the air velocity, including triangle-wing-fin-tube, convex-fin-tube and plat-fin-tube via numerical simulation and experiment in this paper.

\section{Experimental apparatus and methods}

\subsection{Test facility}

Fig.1 is an experimental system diagram of heat transfer characteristics of fin-tube cooler. A cooler unit consists of with aluminum fins and copper pipes. Fig. 2 is the physical sample of cooler.

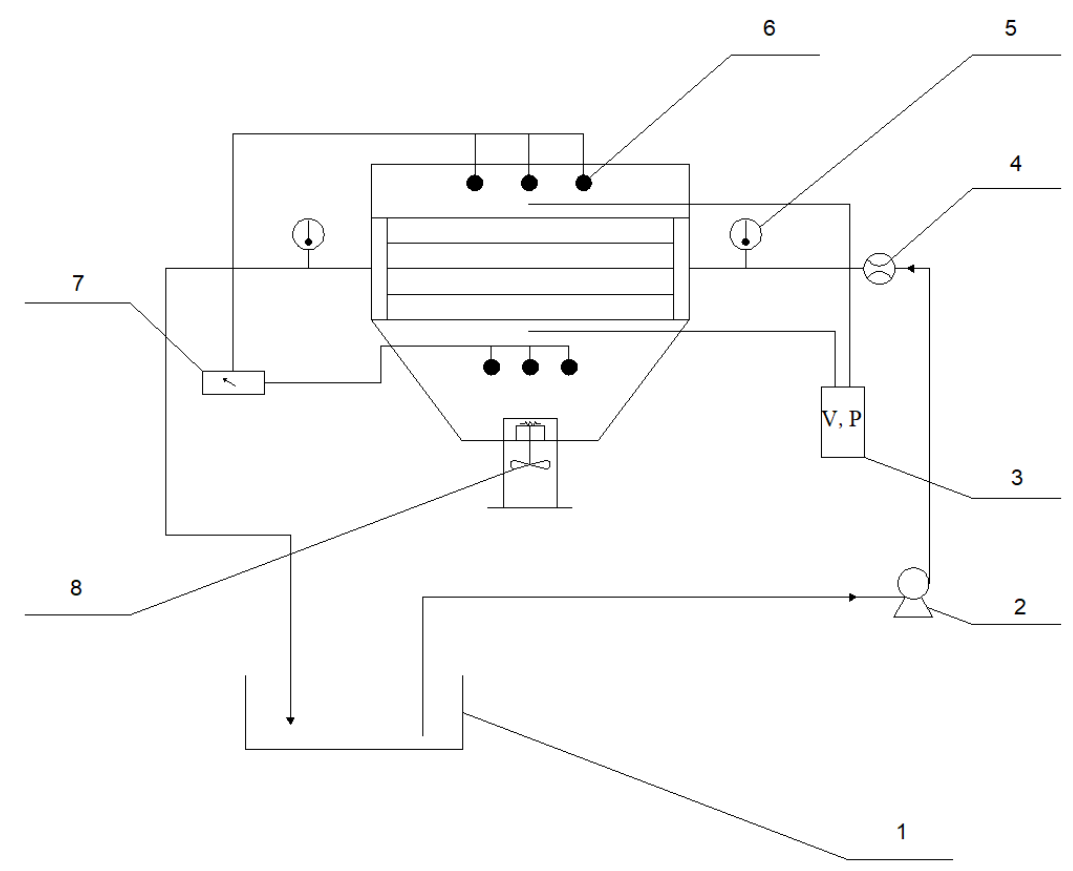

1-Circulating water tank, 2-Water pump, 3-Anemometer, 4-Rotameter, 5、6-Thermocouple temperature sensor, 7- K-type multi-channel temperature inspection instrument, 8-Warm air fan

Fig 1: Flow chart of experimental scheme of heat transfer performance 


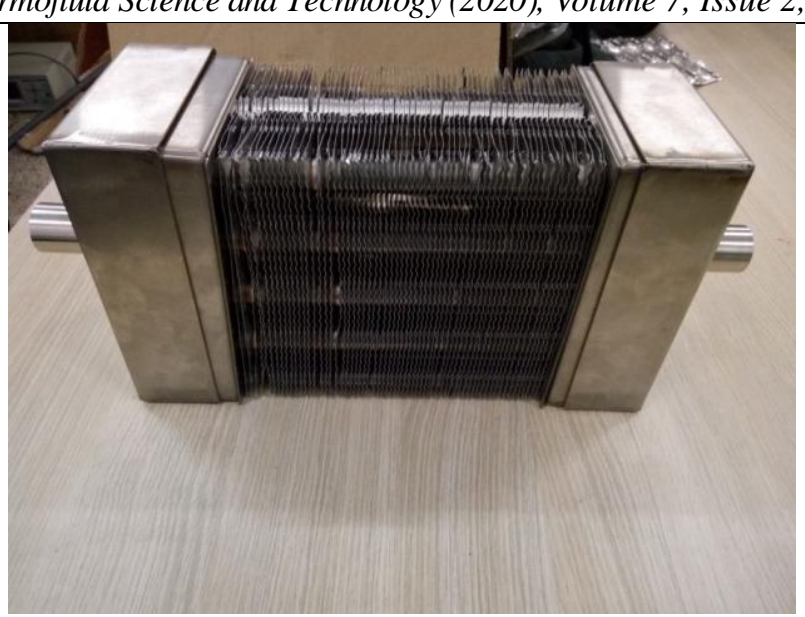

Fig 2: The physical sample of cooler

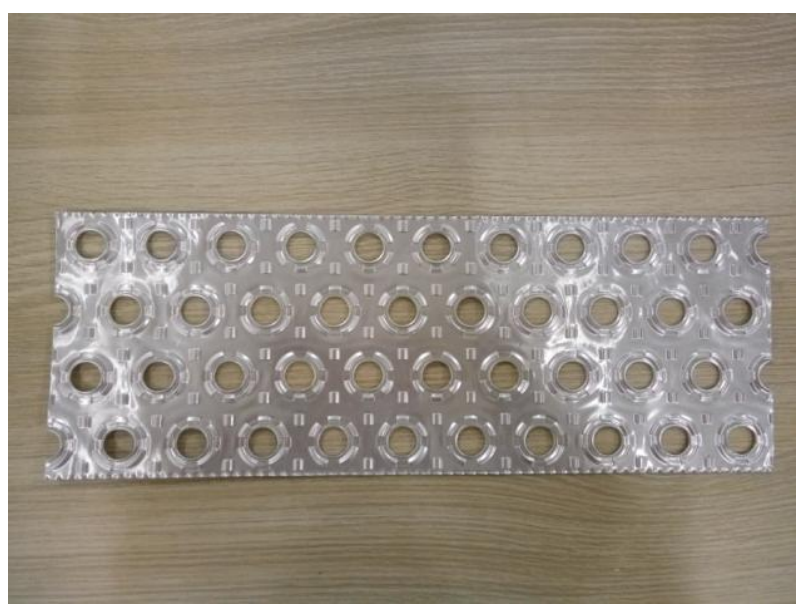

Fig 3: Picture of convex-fin

The experimental system is mainly composed of fin-tube cooler test pieces, cooling water circulation system, hot air system and test system. When it working, ambient air heated by PTC (Positive Temperature Coefficient) ceramic heater and then sent to test piece inlet via duct fan. The normal temperature water sucked by water pump and transport to cooler inlet. Finally, heat transfer between cooling water with hot air in cooler, then they both discharged via fin-tubes and air duct respectively, cooled hot air directly enters atmosphere while hot water enters circulating water tank and enters the next cycle. During the experiment, the coolers' inlet wind speed was measured by the high-precision electronic anemometer. The inlet and outlet temperatures of air in air duct and cooling water in stainless steel braided hose are measured by K-type multi-channel temperature inspection instrument. The pressure drops of cooler in the air duct is measured by $\mathrm{U}$-type pressure gauge.

\subsection{Data processing}

The heat transfer coefficient $K$ of the integral plate-fin cooler is calculated by equation (1):

$$
K=\frac{Q}{A \cdot \Delta t_{m}}
$$


Yuzhu Zhou et al.

International Journal of Thermofluid Science and Technology (2020), Volume 7, Issue 2, Paper No. 070204

Where $Q$ is heat transfer per unit time, which can be calculated by equation (2), $A$ is heat transfer area, $\Delta t_{m}$ is logarithmic mean temperature difference, which can be calculated by equation (3).

$$
\begin{aligned}
& Q=q_{m 1} c_{p 1}\left(T_{1}-T_{2}\right)=q_{m 2} c_{p 2}\left(t_{2}-t_{1}\right) \\
& \Delta t_{m}=\frac{\Delta t_{2}-\Delta t_{1}}{\ln \frac{\Delta t_{2}}{\Delta t_{1}}}
\end{aligned}
$$

Where $q_{m 1}, q_{m 2}$ is mass flow of hot and cold fluids, $c_{p 1}, c_{p 2}$ is average constant pressure specific heat capacity of hot and cold fluids, $T_{1}, T_{2}$ is inlet and outlet temperature of hot fluid, $t_{1}, t_{2}$ is inlet and outlet temperature of cold fluid.

\subsection{Uncertainty analysis}

An uncertainty analysis for the measurement is carried out using the method developed by Moffat [11], which is defined as:

$$
e=\sqrt{\left(\sum_{i=1}^{n} \frac{\partial W}{\partial x_{i}} \Delta x_{i}^{2}\right.}
$$

where $W$ is the estimated parameter, $x$ and $\Delta x$ are the independent parameter and its associated uncertainty, respectively. In Table 1, the main specifications and calculated uncertainties of the measurement instruments and sensors are listed. The uncertainty of measured heat transfer coefficient is thus estimated to be $5.0 \%$.

\begin{tabular}{cccc}
\hline & Table 1 : Specifications of measurement uncertainties & \\
\hline Parameter & Instrument (Type) & Range & Uncertainty \\
& Four-way temperature inspection & $0-9999^{\circ} \mathrm{C}$ & $\pm 0.2 \%$ \\
Temperature & instrument (A0Z80U) & $0-1300^{\circ} \mathrm{C}$ & $\pm 0.75 \%$ \\
& K-type thermocouple & $0-100 \mathrm{kV}$ & $\pm 5 \%$ \\
\hline \multirow{2}{*}{ Mass flow } & Digital rotor flowmeter(FL-10) & & \\
\hline
\end{tabular}

\section{Numerical methods}

\subsection{Geometric model}

In order to strengthen the cooling efficiency of the motor cooler, it is often to increase the heat exchange area by adding flat fins. This is an effective way to strengthen heat exchange with fins. The structure is shown in Fig.4 (c). However, because the airflow will smoothly pass through the fins, a stable boundary layer is formed at the fins and the circular tube boundary, so that the heat transfer of the fluid at the boundary generates a large thermal resistance. In order to cut the thin boundary layer and obtain better convective heat transfer effects, two new type of finned-tube motor cooler with triangle-wing-fin and convex-fin are proposed in this paper. The structures are shown in Fig.4 (a) and (b). 
Yuzhu Zhou et al.

International Journal of Thermofluid Science and Technology (2020), Volume 7, Issue 2, Paper No. 070204

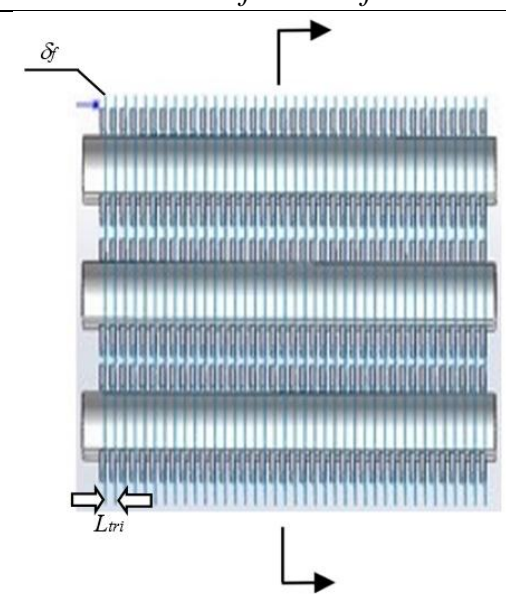

(a)Main view of tube bundle
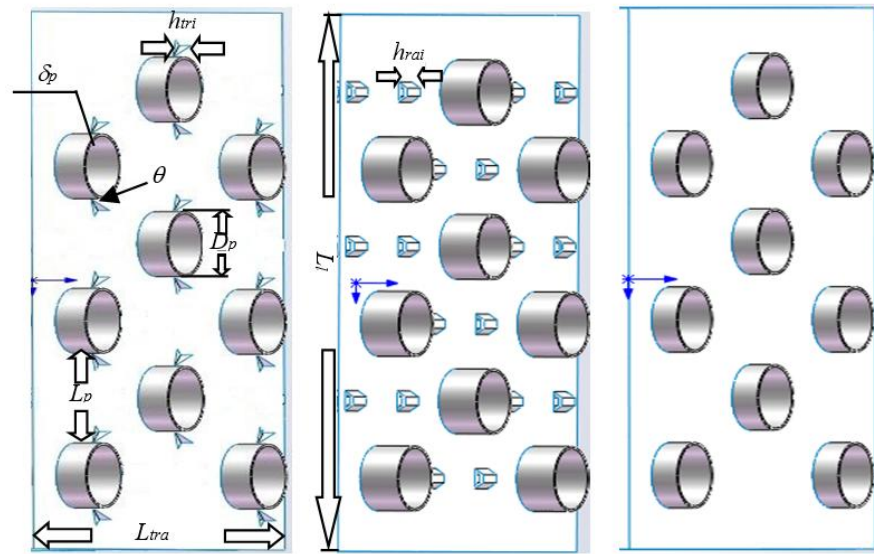

(b) Triangle-wing-fin

(c) Convex-fin

(d) Flat-fin

Fig 4: Geometric model diagram of three kinds fins

\begin{tabular}{|c|cc|}
\hline \multicolumn{3}{c}{ Table 2: Geometric model size } \\
\hline Name & Symbol & Size \\
\hline Transverse length of fins & $L_{t r a}$ & $132 \mathrm{~mm}$ \\
\hline Longitudinal length of fins & $L_{l}$ & $110 \mathrm{~mm}$ \\
\hline Spacing of heat exchange tubes & $L_{p}$ & $38 \mathrm{~mm}$ \\
\hline Outer diameter of heat exchange tube & $D_{p}$ & $16 \mathrm{~mm}$ \\
\hline Wall thickness of heat exchange tube & $\delta_{p}$ & $1 \mathrm{~mm}$ \\
\hline Spacing of fins & $L_{t r i}$ & $4 \mathrm{~mm}$ \\
\hline Thickness of fins & $\delta_{f}$ & $0.2 \mathrm{~mm}$ \\
\hline Angle of triangle-wing & $\theta$ & $30^{\circ}$ \\
\hline Height of triangle-wing & $h_{t r i}$ & $2 \mathrm{~mm}$ \\
\hline Height of convex plat & $h_{r a i}$ & $2 \mathrm{~mm}$ \\
\hline
\end{tabular}

\subsection{Basic assumptions}

Because the movement of fluid in the motor cooler is a complex process, simplifying the calculation model properly can obtain more accurate prediction value and reduce the calculation resources. The following assumptions are made in this paper:

(1) The object of this study is the air side of the cooling unit, the air inlet temperature is $343 \mathrm{~K}$, ignoring the influence of radiation heat transfer.

(2) It is considered that the intercepted cooling unit is the stable section of the actual working condition of the cooler.

(3) The influence of the axial heat transfer of the heat exchange tube and the reverse heat transfer between the tube banks is not considered, only the heat transfer through the fins in the vertical direction of the heat exchange tube is considered.

\subsection{Governing equations}


Yuzhu Zhou et al.

International Journal of Thermofluid Science and Technology (2020), Volume 7, Issue 2, Paper No. 070204

The governing equations are three-dimensional incompressible, steady-state, constant physical and turbulent flow equations.

Mass conservation equation:

$\frac{\partial}{\partial x_{i}}\left(\rho u_{i}\right)=0$

Momentum conservation equation:

$\frac{\partial}{\partial x_{j}}\left(\rho u_{i} u_{j}\right)=-\frac{\partial P}{\partial x_{i}}+\frac{\partial}{\partial x_{j}}\left[\mu\left(\frac{\partial u_{i}}{\partial x_{j}}+\frac{\partial u_{j}}{\partial x_{i}}-\frac{2}{3} \delta_{i j} \frac{\partial u_{i}}{\partial x_{i}}\right)\right]+\frac{\partial}{\partial x_{j}}\left(-\rho \vec{u}_{i} \vec{u}_{j}^{\prime \prime}\right)$

Energy conservation equation:

$\operatorname{div}(u T)=\operatorname{div}\left(\frac{\lambda}{\rho c_{p}} \operatorname{grad} T\right)+\frac{S_{T}}{\rho}$

The turbulent transport equation can be expressed as the turbulent energy transport equation (8) and the energy dissipation transport equation (9):

$\frac{\partial}{\partial x_{j}}\left(\rho u_{j} \frac{\partial k}{\partial x_{j}}-\left(\mu+\frac{\mu_{\tau}}{\sigma_{k}}\right) \frac{\partial k}{\partial x_{j}}\right)=\tau_{t i j} S_{i j}-\rho \varepsilon+\varphi_{k}$

$\frac{\partial}{\partial x_{j}}\left(\rho u_{j} \varepsilon-\left(\mu+\frac{\mu_{\tau}}{\sigma_{\varepsilon}}\right) \frac{\partial \varepsilon}{\partial x_{j}}\right)=c_{\varepsilon 1} \frac{\varepsilon}{k} \tau_{t i j} S_{i j}-c_{\varepsilon 2} f_{2} \rho \frac{\varepsilon^{2}}{k}+\varphi_{\varepsilon}$

The right-hand term in Equation (8) represents the generated term, the dissipative term, and the wall term. The values of each constant are as follows:

$c_{\mu}=0.09, c_{\varepsilon 1}=1.45, c_{\varepsilon 2}=1.92, \sigma_{\mathrm{k}}=1.0, \sigma_{\varepsilon}=1.3$

\subsection{Grid independence}

Because of triangle-wing and convex-fin-tube on the cooler, the model is more complex, the unstructured grid is selected for division. Fig.5 is the internal grid map of the hidden external watershed.

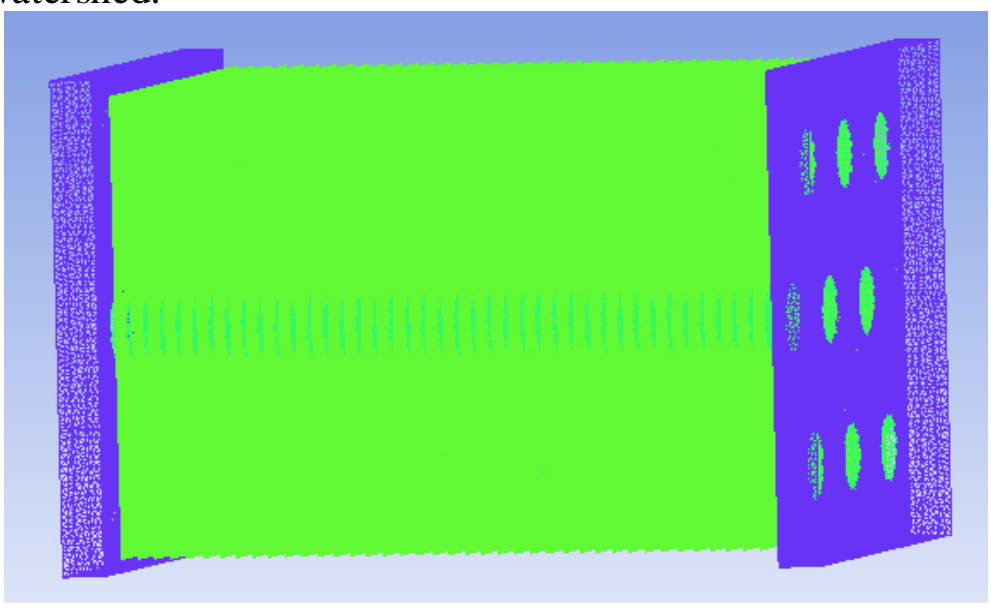

Fig 5: Mesh generation 


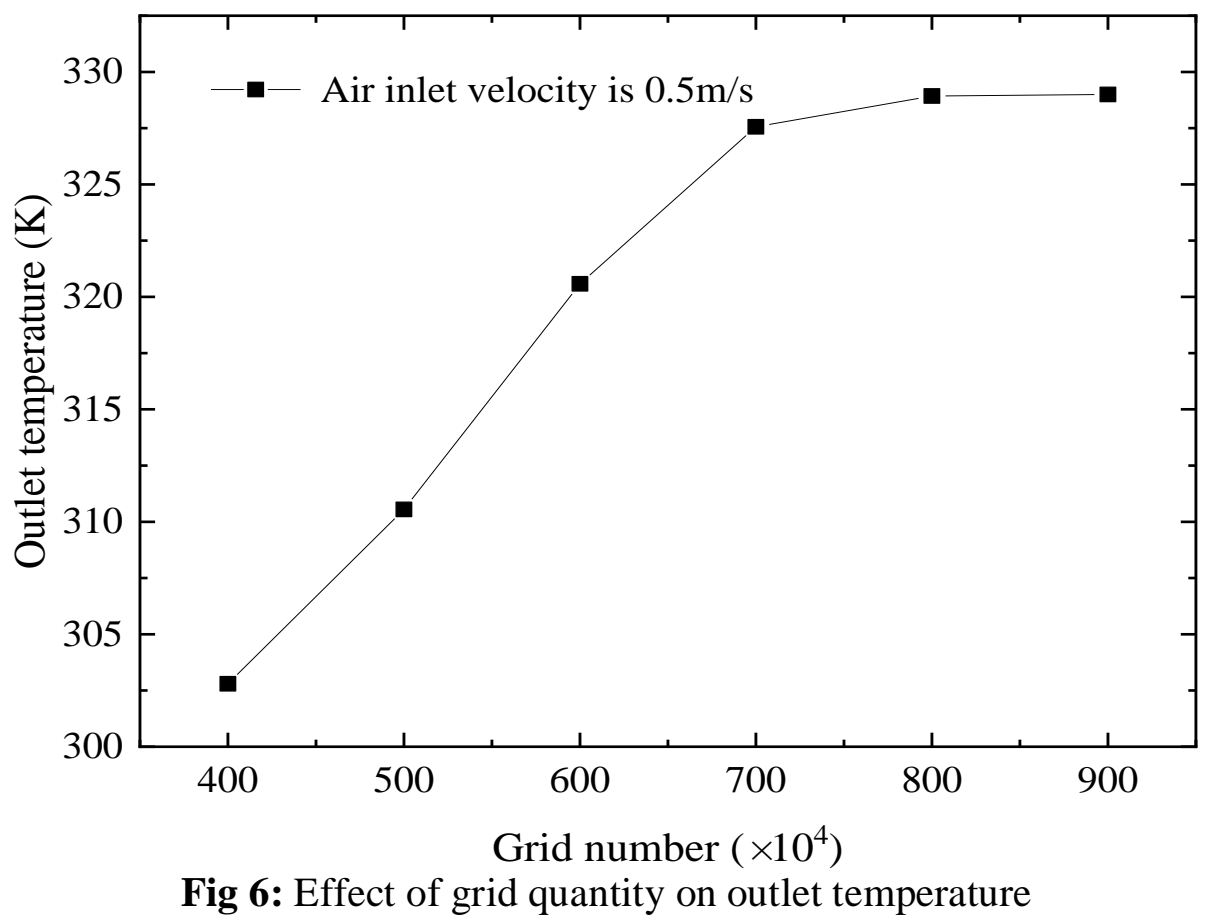

In order to verify the feasibility of grid, the models with grid number of 4, 5, 6, 7, 8 and 9 million used for checked respectively. The results are shown in Fig.6. When the grid number reaches 7 million, the temperature at outlet of cooler hardly changes when grid number increased. Considering the accuracy of the calculation and the computing resources, the final calculation is performed with a grid number of 7 million.

\subsection{Boundary conditions}

All entrance boundary conditions are set as velocity inlet, the air-side and cooling water inlet velocity range of 0.5 to $5 \mathrm{~m} / \mathrm{s}$ and 0.2 to $1.4 \mathrm{~m} / \mathrm{s}$, respectively. The initial inlet temperature of hot air and cooling water are $343 \mathrm{~K}$ and $293 \mathrm{~K}$. The outlet boundary conditions are set as pressure which equals a standard atmospheric. All walls are coupled wall with no slip conditions, and the fin thickness is set to $0.2 \mathrm{~mm}$. The fin material is set to aluminum, and the heat exchange tube material is copper.

\section{Results and Discussions}

\subsection{Numerical method verification}

Fig.7 shows variance between experiment with simulation of average heat transfer coefficient of the convex-fin-tube cooler with a fin pitch of $2.5 \mathrm{~mm}$, which can be seen the trend of experiment and simulation are basically the same. When air inlet velocity increased, the average heat transfer coefficient also improved. Especially, once the air inlet speed is reached $2 \mathrm{~m} / \mathrm{s}$, the growth rate of the average heat transfer coefficient will become smooth. When the speed is less than $2 \mathrm{~m} / \mathrm{s}$, the experimental value is close to the simulated value. When the speed is greater than $2 \mathrm{~m} / \mathrm{s}$, the difference between the two is obvious, it can be attributed to a fact that the degree of airflows' turbulence in the channel increases with velocities improve. The velocity at $2 \mathrm{~m} / \mathrm{s}$ is the boundary point of turbulence's degree. When the velocity is lower than this, the flow is in the stage of development. On the contrary, once 
Yuzhu Zhou et al.

International Journal of Thermofluid Science and Technology (2020), Volume 7, Issue 2, Paper No. 070204

velocity is exceeded that, the flow will become sufficiently turbulent. Obviously, the accuracy of model's estimated motion will be affected by the turbulence of airflow. Although the simulation results will have some minor variances when the airflow reaches strong turbulence, fortunately, the maximum error is $10 \%$ which can be accepted. The numerical method is feasible and has high accuracy.

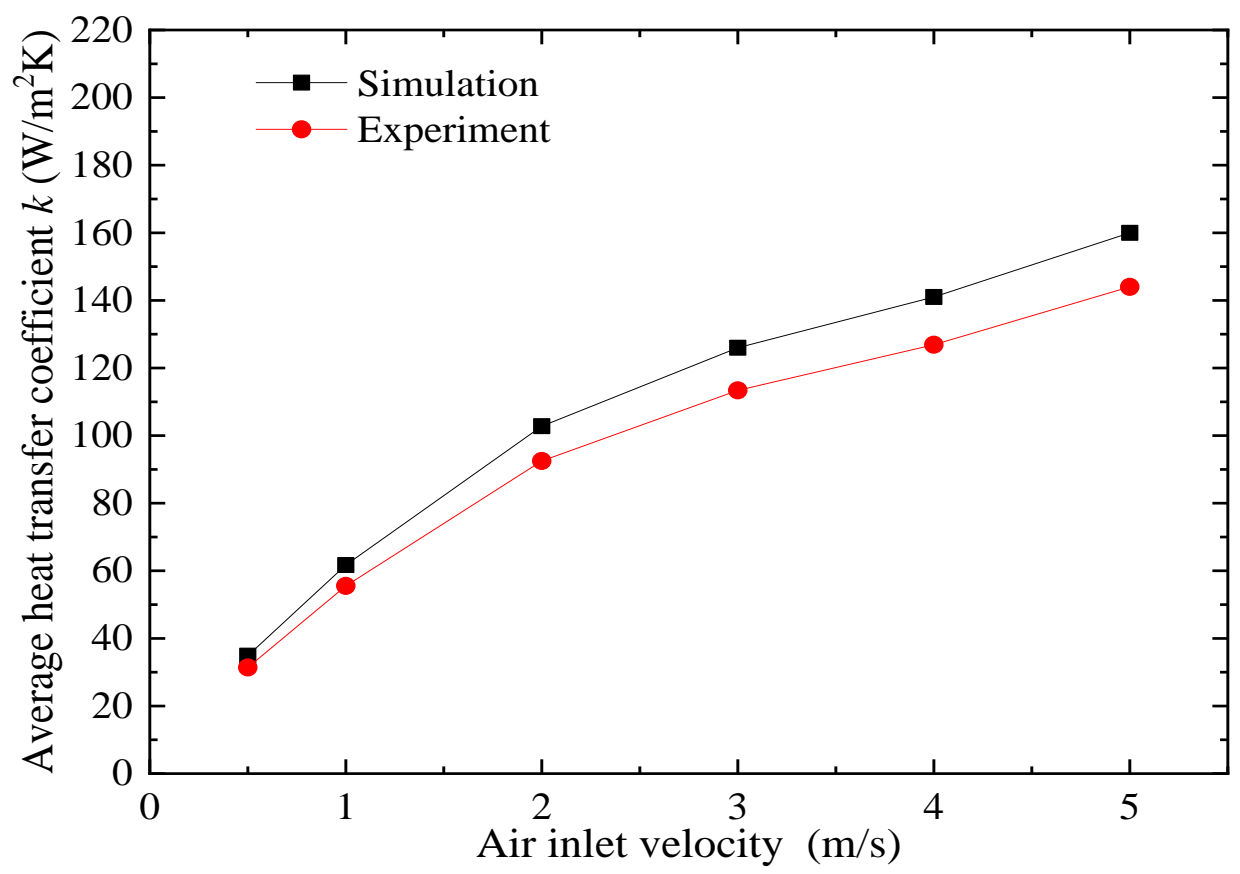

Fig 7: Comparison of average heat transfer coefficient between experiment and simulation

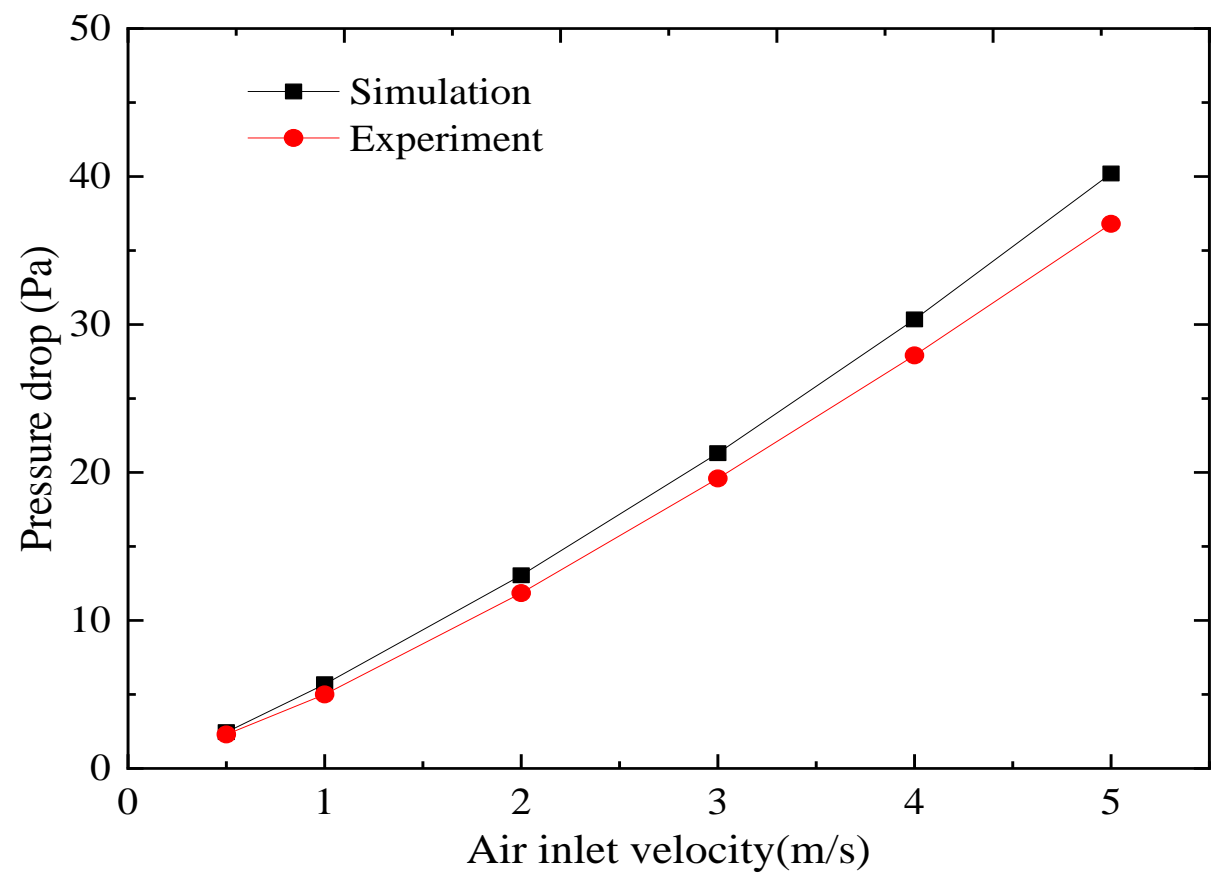

Fig 8: Comparison with pressure drop between experiment and simulation

Fig. 8 gives a comparison between experiment and simulation of pressure drop. It can be known that experiment change of pressure drop is basically consistent with simulation. As 
Yuzhu Zhou et al.

International Journal of Thermofluid Science and Technology (2020), Volume 7, Issue 2, Paper No. 070204

the air inlet velocity increased, the curve gradually approaches a one-dimensional linear curve. The difference between the two is relatively small, the maximum error is $8 \%$. It shows that the k- $\varepsilon$ turbulence model is more accurate in predicting the change trend of the pressure drop of the cooler.

\subsection{Flow field analysis}

Fig.9 is the overall temperature distribution of the cooler when air inlet speed at $1 \mathrm{~m} / \mathrm{s}$. The triangle-wing and convex-fin can improve heat transfer at the leeward side of tube which named heat exchange dead zone and reduce its area, so heat exchange efficiency of cooler can be improved. When hot air passes over the triangle-wing fin, the temperature field distribution is very uneven and chaotic, especially at the exit, the temperature field in the heat exchange area at the leeward side of the cooling water pipe is disturbed. This shows that strong disturbances generated when hot air flows through the surface of the fins with triangle-wing, it can extend the time for indirect convective heat exchange between hot air and cooling water. Different from the former, when hot air is swept over the flat fins, in addition to generating a certain cylindrical flow, the temperature field distribution of the entire section is relatively uniform. The temperature is lower near the pipe wall, so the temperature field near the cooling water pipe is delaminated. The surface of the flat fin is relatively smooth, and the disturbance effect on the hot air flow is relatively small. Therefore, the air flow stays in the heat exchange area for a short time, making the heat exchange process relatively slow.

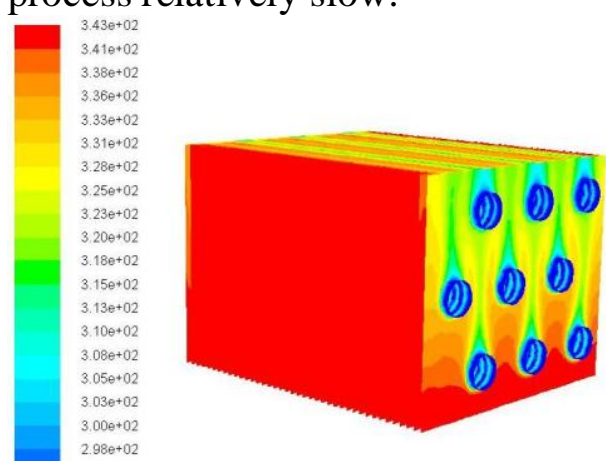

(a) Flat-fin

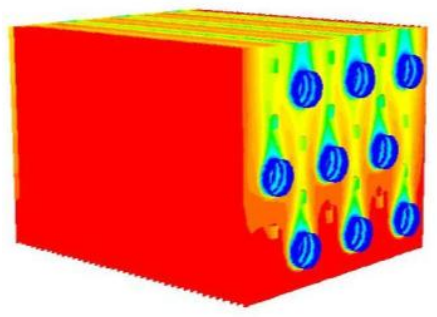

(b) Convex-fin

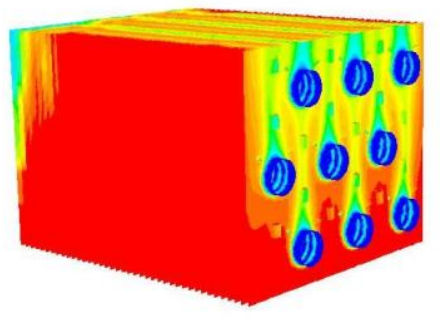

(c) Triangle-wing-fin

Fig 9: The overall temperature distribution of the cooler

\subsection{Heat transfer characteristics}

Fig.10 shows the relationship between average heat transfer coefficient and air inlet speed. On the whole, the average heat transfer coefficient of three fin-tubes will increase with air velocity improve, and it show a trend of quadratic function, but the growth rate is not same. At low Reynolds numbers, the heat transfer coefficients are relatively close. As the Reynolds number increases, the heat transfer coefficient of the triangle-wing-fin increases more than others. It is can be attributed to these reasons; on the one hand, air can be driven more disturbance on the flat plate which cut the boundary layer formed on the surface, it is easier for the air near the plate to cross the boundary layer and reach the fins' surface, thereby improving the transfer of heat flow to the air side. This trend is also reflected in convex-fin. In addition, the triangle-wing-fin can destroy the boundary layer near the round 
Yuzhu Zhou et al.

International Journal of Thermofluid Science and Technology (2020), Volume 7, Issue 2, Paper No. 070204

tube. Air will form cylindrical flow on the tubes' surface without triangle-fin. The non-condensable gas will cause air have to cost more power to reach tube. The triangle-wing-fin causes the airflow layer on the surface to be stripped, and the tendency of the cylinder to flow around is destroyed, which makes the air can easily to reach the tubes, which further strengthens the heat transfer efficiency of the entire cooler.

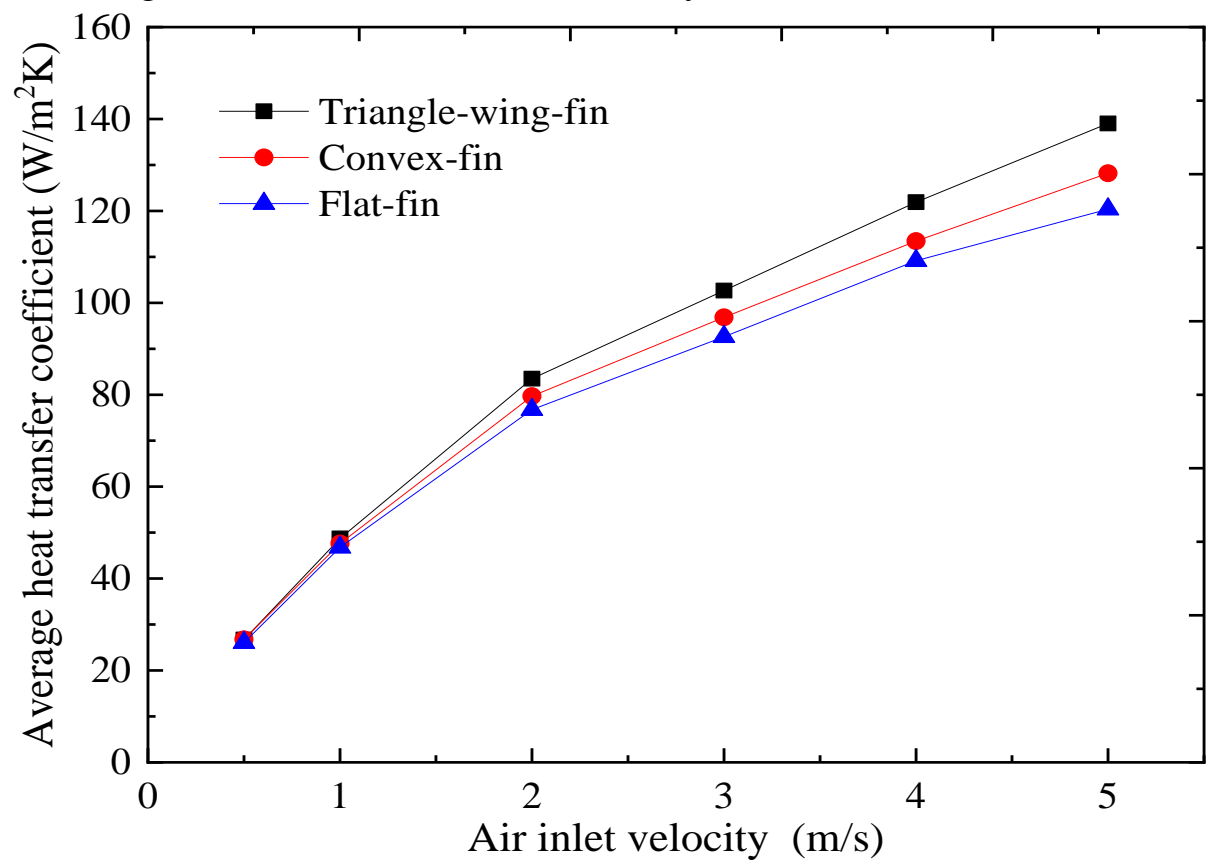

Fig 10: The relation curve of average heat transfer coefficient and air inlet velocity

In addition to the influence of the air inlet speed, the average heat transfer coefficient is also related to the spacing between the fins. Fig.11 reveals the relationship between average heat transfer coefficients and two fin pitches based on the convex-fin. It can be found that average heat transfer coefficients of the two fin spacings are basically the same in the overall trend, but the average heat transfer coefficient of the $2.5 \mathrm{~mm}$ fin spacing is significantly higher than that of the $4 \mathrm{~mm}$ fin spacing. When the distance between the fins is small, the entire flow path of the cooler is divided into several smaller distances by the fins. When air flows through the flow path, the contact area with the fins is relatively increased, which improves the heat exchange effect. When the distance between the fins increases, the boundary layers between the fins are independent of each other. As the distance gradually increases, the area ratio of the base pipe surface area with a relatively small heat transfer coefficient also gradually increases. Therefore, the average heat transfer coefficient also decreases. 


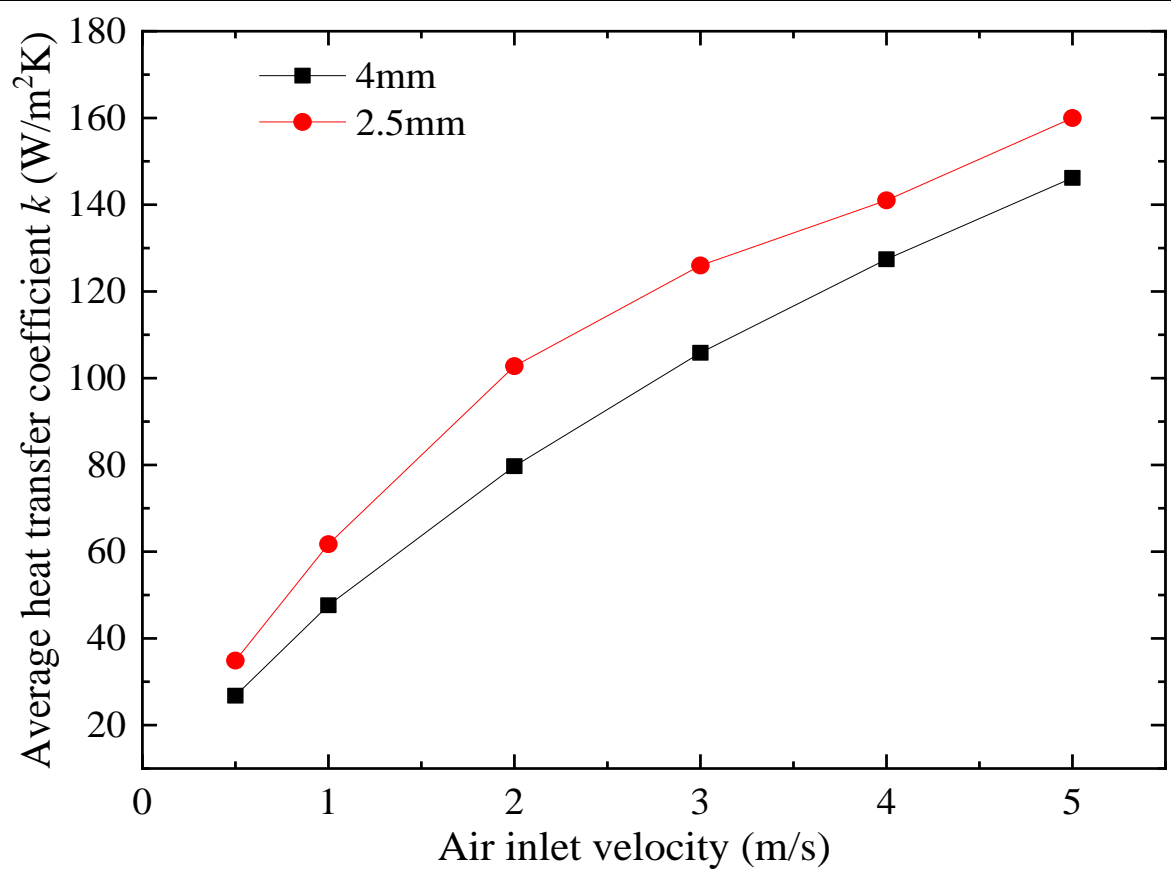

Fig 11: The relation curve of average heat transfer coefficient with different fin spacing

Fig.12 shows the effect of air speed on the overall cooler resistance. Regardless of the structure, the pressure drop at the inlet and outlet of the cooler will increase with the increase of air velocity. This trend appears as a quadratic function with a positive quadratic coefficient, which indicates that the more the Reynolds number at the inlet increases, the more additional pressure loss. At the same air inlet speed, the pressure drop of triangle-wing-fin is the largest, and the pressure drop of flat-fin is the lowest. On the one hand, the lateral interception of the triangle-wing-fin makes the circulation area of air from entering the cooler to the final exit smaller, which creates additional form resistance; On the other hand, triangle-wing-fin strips off the fluid flowing around the cylinder, and also interferes with the air flow on the flat surface. These additional disturbances cause the local air velocity to increase sharply, which will generate additional flow resistance. The structure of convex-fin is simpler than triangle-wing-fin, but it is more complicated than flat-fin. Therefore, the pressure drop of the convex-fin is smaller than that of triangle-wing-fin and larger than that of flat-fin. 


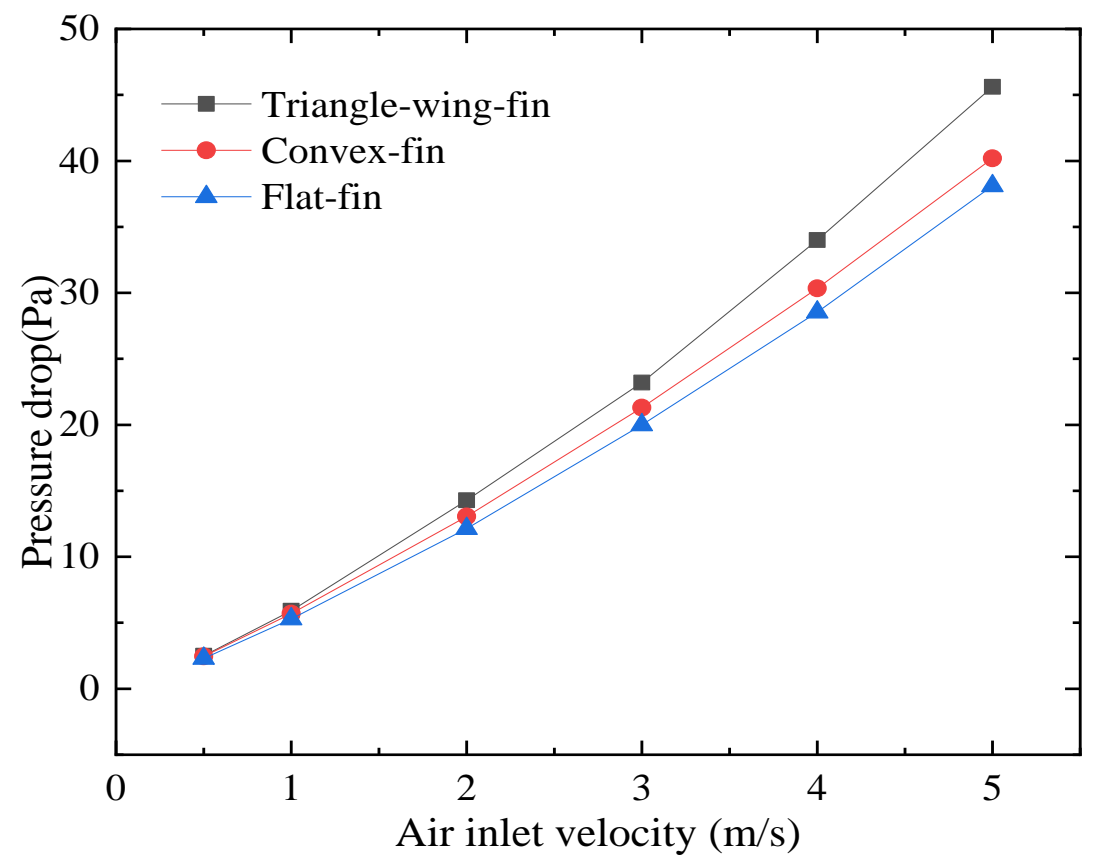

Fig 12: The relation curve of air inlet velocity and pressure drop

Fig.13 shows effect of water velocity in the heat transfer coefficient. As can be seen from the figure that the heat transfer coefficient grows with the rising water velocity at relatively low speed. However, as the water velocity rises above $0.4 \mathrm{~m} / \mathrm{s}$, the heat tranfer coefficient remains nearly constant. That's because the flow state is at the transition zone between the laminar flow and fully developed turbulence, the rising water velocity will make the boundary layer thinner, which leads to the significantly improving of the heat transfer coefficient. With the further increasing water velocity, above $0.4 \mathrm{~m} / \mathrm{s}$, the flow reachs the stage of fully developed turbulence, in which the obvious boundary layer resulted by fluid retention no longer exist. In other words, the further increasing water velocity will not improve the heat transfer coefficient significantly. 


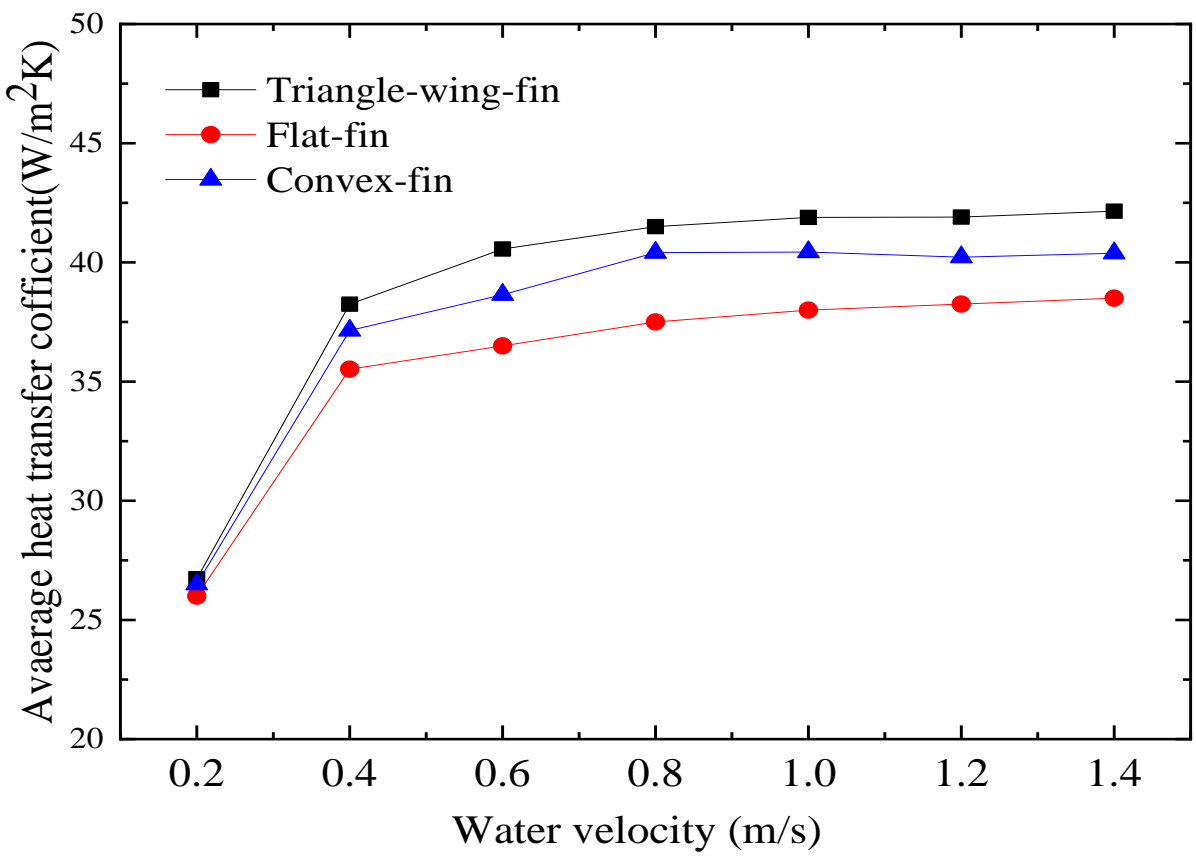

Fig 13: The relation curve of water inlet velocity and heat transfer coefficient

\section{Conclusion}

Analysis of coolers' overall performance with various plate-fin-tubes was investigated through experiment and numerical simulation. The conclusions are summarized as follows:

- The k- $\varepsilon$ turbulence model can accurately predict the flow and heat transfer characteristics of plate-fin coolers. The maximum error of the heat transfer coefficient is $10 \%$, and the maximum error of the pressure drop is $8 \%$.

- Comparing with flat-fin, there is more disturbance product when hot air passing by fin-tube with triangle-wing or convex-fin. So, in the latter, the air stays longer which introduce to more heat transfer.

- In terms of heat coefficient, triangle-wing fin shows better performance. However, a large of pressure drop drive form geometric resistance. Considering resistance and heat transfer coefficient together, the convex-fin may be the most optimal structure applied for cooler.

\section{Acknowledgements}

This research is funded by the Jiangsu Provincial Major Projects of Natural Science Research for the Institution of Higher Education (Grant No. 18KJA480002), Projects of Jiangsu Provincial Six Talent Peaks (Grant No. GDZB-CXTD-001), Key R\&D Project of Changzhou City in 2018 (Grant No. CE20185036), Key R\&D Program of Dantu District of Zhenjiang City--Industry Prospect and Common Key Technologies (Grant No. GY2018006). They are gratefully acknowledged. 
Yuzhu Zhou et al.

International Journal of Thermofluid Science and Technology (2020), Volume 7, Issue 2, Paper No. 070204

\section{References}

[1] E. Galloni, P. Parisi, F. Marignetti, G. Volpe, CFD analyses of a radial fan for electric motor cooling, Therm. Sci. Eng. Pro. 8 (2018) 470-476 .

[2] B. Melka, J. Smolka, J. Hetmanczyk, P. Lasek, Numerical and experimental analysis of heat dissipation intensification from electric motor, Energy 182 (2019) 269-279.

[3] Mohammad Sikindar Baba, A.V. Sita Rama Raju, M. Bhagvanth Rao, Heat transfer enhancement and pressure drop of $\mathrm{Fe}_{3} \mathrm{O}_{4}$-water nanofluid in a double tube counter flow heat exchanger with internal longitudinal fins, Case Studies in Thermal Engineering 12 (2018) 600-607.

[4] Xiaoqin Liu, Jianlin Yu, Guang Yan. A numerical study on the air-side heat transfer of perforated finned-tube heat exchangers with large fin pitches. Int. J.Heat Mass Transf. 100 (2016) 199-207.

[5] Sachin Gupta,Aditya Roy,Arvind Gupta. Computer-aided engineering analysis for the performance augmentation of a fin-tube heat exchanger using vortex generator. Concurrent Engineering,2020,28(1).

[6] Jong Hwi Lee,Jong-Hyeon Shin,Se-Myong Chang,Taegee Min. Numerical Analysis on Natural Convection Heat Transfer in a Single Circular Fin-Tube Heat Exchanger (Part 1): Numerical Method. Entropy,2020,22(3).

[7] Jong Hwi Lee,Young Woo Son,Se-Myong Chang. Numerical Analysis on Natural Convection Heat Transfer in a Single Circular Fin-Tube Heat Exchanger (Part 2): Correlations for Limiting Cases. Entropy,2020,22(3).

[8] Jiyang Li, Chaobin Dang, Eiji Hihara. Heat transfer enhancement in a parallel, finless heat exchanger using a longitudinal vortex generator, Part A: Numerical investigation, Int.J. Heat Mass Transf. 128 (2019) 87-97.

[9] Jiyang Li, Chaobin Dang, Eiji Hihara. Heat transfer enhancement in a parallel, finless heat exchanger using a longitudinal vortex generator, Part B: Experimental investigation on the performance of finless and fin-tube heat exchangers. Int.J. Heat Mass Transf. 128 (2019) $66-75$.

[10] Mohd Fahmi Md Salleh, Hussein A. Mohammed, Mazlan A. Wahid, Thermal and hydraulic characteristics of trapezoidal winglet across fin-and-tube heat exchanger (FTHE), Appl. Thermal Eng. 149 (2019) 1379-1393.

[11] V. Glazar, A. Trp, K. Lenic, Numerical study of heat transfer and analysis of optimal fin pitch in a wavy fin-and-tube heat exchanger, Heat Transfer Eng. 33 (2012) 88-96.

[12] D.H. Park, D.B. Lee, E.R. Seo, Y.J. Park, Study on the heat transfer and fluid flow characteristics in V-shaped corrugated composite fin, Appl. Thermal Eng. 102 (2016) 293-301.

[13] Z. Carija, B. Franković, M. Percic, M. Cavrak, Heat transfer analysis of fin-and-tube heat exchangers with flat and louvered fin geometries, Int. J. Refrig. 45 (2014) 160-167.

[14] H. Li, H. Wang, M. Yao, L. Zhang, H. Gu, J. Nie, PIV and thermal-vision experimental and numerical investigation on the airside performance of slotted fin surfaces, Int. J. Heat Mass Transf. 82 (2015) 568-580.

[15] S. Wongwises, Y. Chokeman, Effect of fin pitch and number of tube rows on the air side performance of herringbone wavy fin and tube heat exchangers, Energy Convers. Manag. 46 (2005) 2216-2231. 
Yuzhu Zhou et al.

International Journal of Thermofluid Science and Technology (2020), Volume 7, Issue 2, Paper No. 070204

[16] A. Gholami, M.A. Wahid, H.A. Mohammed, Thermal-hydraulic performance of finand-oval tube compact heat exchangers with innovative design of corrugated fin patterns, Int. J. Heat Mass Transf. 106 (2017) 573-592.

[17] A. Gholami, M.A. Wahid, M.M. Sies, M.Y.M. Fairus, N. Kamaruzaman, A. Saat, A.-M. Z., H.A. Mohammed, M.M. Rahman, Investigation on heat transfer enhancement in a corrugated fin-and-tube compact heat exchanger, J. Teknol. 78 (2016) 7-13.

[18] Fei-Long Wang, Song-Zhen Tang. Heat transfer and fouling performance of finned tube heat exchangers: Experimentation via on line monitoring, Fuel 236 (2019) 949-959.

[19] Pengfei Wang,Jin Jiang,Shunyang Li,Xiangyu Luo,Shaojie Wang,Wensheng Zhao. An investigation of influence factor including different tube bundles on inclined elliptical fin-tube heat exchanger. Int.J. Heat Mass Transf.2019,142.

[20] M.J. Li, H. Zhang, J. Zhang, Y.T. Mu, E. Tian, D. Dan, X.D. Zhang, W.Q. Tao, Experimental and numerical study and comparison of performance for wavy fin and a plain fin with radiantly arranged winglets around each tube in fin-and-tube heat exchangers, Appl. Thermal Eng.133 (2018) 298-307.

[21] Nae-Hyun Kim. Airside Performance of Fin-and-Tube Heat Exchangers Having Nonrepeating Nonsymmetrical Slit Fins under Wet Condition. International Journal of Air-Conditioning and Refrigeration,2019,27(04).

[22] Ephraïm Toubiana, Rémi Gautier, Daniel Bougearda, Serge Russeil. Large Eddy Simulation of transitional flows in an elliptical finned-tube heat exchanger, Int.J.Thermal Sci.144 (2019) $158-172$. 